\title{
Age-Related Decline in Cardiorespiratory Fitness among Career Firefighters: Modification by Physical Activity and Adiposity
}

\author{
Dorothee M. Baur, ${ }^{1,2}$ Costas A. Christophi, ${ }^{1,3}$ E. Francis Cook, ${ }^{4}$ and Stefanos N. Kales ${ }^{1,2}$ \\ ${ }^{1}$ Environmental and Occupational Medicine and Epidemiology (EOME), Department of Environmental Health, \\ Harvard School of Public Health, Boston, MA 02115, USA \\ ${ }^{2}$ Employee Health and Industrial Medicine, Harvard Medical School and The Cambridge Health Alliance, 1493 Cambridge Street, \\ Macht 427, Cambridge, MA 02139, USA \\ ${ }^{3}$ Cyprus International Institute for Environmental and Public Health in Association with Harvard School of Public Health, \\ Cyprus University of Technology, 3036 Limassol, Cyprus \\ ${ }^{4}$ Division of General Internal Medicine and Primary Care, Department of Medicine, Brigham and Women's Hospital and \\ Harvard Medical School, Boston, MA 02115, USA
}

Correspondence should be addressed to Stefanos N. Kales, skales@hsph.harvard.edu

Received 10 January 2012; Accepted 9 March 2012

Academic Editor: Panagiota Nota Klentrou

Copyright (C) 2012 Dorothee M. Baur et al. This is an open access article distributed under the Creative Commons Attribution License, which permits unrestricted use, distribution, and reproduction in any medium, provided the original work is properly cited.

\begin{abstract}
Firefighting is a very hazardous occupation, and strenuous fire duties require high levels of physical fitness. In the general adult population, cardiorespiratory fitness (CRF) declines with aging. We sought to investigate the effect of increasing age on CRF in male career firefighters as well as the modifying effects of physical activity and adiposity. We cross-sectionally examined 804 male career firefighters from two Midwestern states. CRF was determined from symptom-limited maximal treadmill exercise testing in metabolic equivalents (METS) following the Bruce protocol. Physical activity self-reports were extracted from responses to a health and lifestyle questionnaire. We found as expected that CRF declines with advancing age; however, the decline is greatly attenuated among leaner firefighters who report more physical activity. Furthermore, in a linear regression model including age, BMI, and variables describing physical activity behaviors, we could predict CRF $\left(R^{2}=0.6286\right)$. The total weekly duration of aerobic exercise as well as the duration of weight lifting sessions both had significant impacts on age-related decline. We conclude that firefighters are more likely to maintain the high levels of CRF needed to safely perform their duties if they engage in frequent exercise and maintain healthy weights.
\end{abstract}

\section{Introduction}

Cardiorespiratory fitness (CRF) declines with aging in the general population. A decrement in $\mathrm{VO}_{2} \max (\mathrm{mL} / \mathrm{kg} / \mathrm{min})$ of about $1.6 \%$ per year in both men and women has been described [1].

Similarly, among untrained individuals, a decline in peak $\mathrm{VO}_{2}$ of $5-10 \%$ per decade of life has been observed [2]. Low $\mathrm{CRF}$ is an important risk factor for the development of obesity, hypertension, and other cardiovascular risk factors as well as coronary heart disease, stroke, loss of independence, and premature mortality [1,3-6], which all increase with advancing age.
Firefighting is known to be a dangerous occupation that is physically demanding. However, inadequate physical activity makes firefighters prone to increased obesity and the metabolic syndrome. Obese firefighters are more susceptible to gain further weight [7] and associated with decline in health status $[7,8]$. We have shown previously that low physical activity and CRF are associated with unfavorable CVD risk profiles as well as higher prevalence of the metabolic syndrome (MetSyn) [9-11].

Many US firefighters receive medical- and physicalabilities testing at the beginning of their professional careers and no subsequent formal reassessment over a 20-30-year work span [12]. Nonetheless, older firefighters are expected 
to be able to perform the same essential job functions as young recruits. Studies of simulated firefighting tasks suggest that the minimum aerobic capacity as measured by oxygen consumption $\left(\mathrm{VO}_{2}\right)$ necessary to safely perform firefighting duties ranges from 33.9 to $45 \mathrm{~mL} / \mathrm{kg} / \mathrm{min}$ (9.7-12.8 METS; $1 \mathrm{MET}=2.5 \mathrm{~mL} / \mathrm{kg} / \mathrm{min})[13,14]$. Therefore, all firefighters should continue to require such relatively high aerobic capacities in order to safely perform their duties regardless of their age. Thus, this study focused on describing CRF across different age categories among professionally active career firefighters. Furthermore, we sought to determine effect modification by physical activity and BMI levels across different ages groups. We hypothesized that leaner and more active firefighters would be more likely to maintain higher aerobic capacities regardless of their age.

\section{Methods}

2.1. Study Population. Eligible male career firefighters, between 18 and 59 years of age were recruited from 10 fire departments in two Midwestern states. Inclusion criteria included having completed a maximal exercise test during the course of a fire department-sponsored medical examination, no work restrictions at examination, and completing the study questionnaire. Excluded subjects failed to meet one or more of the above criteria or had undergone the index exercise tests for the evaluation of symptoms, retirement pensions, disability, and/or exit examinations. The study was approved by the IRB of Harvard School of Public Health and local IRBs as appropriate, and all participants signed an informed consent.

2.2. Assessment of Cardiovascular Risk Factors. Height was measured in the standing position with a clinic stadiometer. Body weight was measured with bare feet and in light clothes on a calibrated scale. Body mass index (BMI) was calculated as the weight in kilograms divided by the square of height in meters. BMI values were also categorized according to the World Health Organization (WHO) classification.

2.3. Cardiorespiratory Fitness. CRF was determined from symptom-limited maximal treadmill exercise testing with ECG monitoring following the Bruce protocol. The participants were encouraged to continue exercise until volitional exhaustion, even after exceeding $85 \%$ of their maximum predicted heart rate defined as 220 minus age. Accordingly, the cohort achieved an average of $97.9 \%$ (SD 6.6) of maximal age-predicted heart rate on these tests. During the exercise test, total treadmill time was recorded in minutes and seconds, and then seconds were converted to fractional minutes by dividing by 60 (e.g., 8 minutes 30 seconds $=8.50$ minutes). CRF was then estimated according to a widely used formula for $\mathrm{VO}_{2}$ max estimation and the Bruce protocol:

$$
\begin{aligned}
\mathrm{VO}_{2} \max (\mathrm{mL} / \mathrm{kg} / \mathrm{min})= & 14.76-(1.379 \times T) \\
& +\left(0.451 \times T^{2}\right)-\left(0.012 \times T^{3}\right),
\end{aligned}
$$

For details on the equation see [15], " $T$ " represents "total exercise tolerance test time" (measured in minutes). Finally, CRF estimates in maximal METS were derived by dividing the $\mathrm{VO}_{2}$ max estimates by 3.5 as per the standard conversion factor.

2.4. Assessment of Physical Activity (PA). Self-reports of PA were extracted from responses to a health and lifestyle questionnaire as previously described from our group [11]. Briefly, consented study participants were given standardized written instructions for completing the multiple-choice survey regarding eating, health, exercise, sleep, and work habits as honestly and as best as they could. They were also informed that the completed questionnaires would be confidential and would not become part of their fire department or medical record. The answers to four selected multiplechoice questions about involvement in sports and exercise were analyzed here: (1) "Most weeks, I exercise .... (include home/work/gym \& elsewhere)"; (2) "Most times that I do cardio or aerobic exercise (e.g., jogging, brisk walking, bike, treadmill, etc.), I do an average of ... each session"; (3) "Most times that I lift weights or do strength training, I do ... on average each session"; (4) "Most times that I exercise, I sweat ... on average each session". Additionally, as described previously an additional variable was calculated to evaluate the combined effects of PA frequency and PA aerobic or cardiosession duration as total weekly aerobic exercise. Each of the six original alternatives in the frequency and aerobic session duration dimension questions received a numerical value equivalent to the middle point of the range of each response choice. Thus, a value of 2.5 times per week was assigned to the frequency response of 2-3 times per week, 3.5 for 3-4 times per week, and so on. The same methodology was applied to the duration responses: 22.5 minutes was assigned for 15 to 30 minutes, 37.5 minutes for durations of 30 to 45 minutes, and so on. Subsequently, the resulting frequency and duration values were multiplied to estimate total weekly exercise in minutes per week.

2.5. Statistical Analysis. Baseline characteristics were described using the mean (SD) in the case of quantitative variables and the frequency in case of categorical variables. Group comparisons were calculated using ANOVA. Linear models and logistic regression models were used to adjust for other variables.

The prediction model for CRF was constructed by dividing the whole dataset at random into a "training" (or analyzed) ( $n=405)$ subgroup and a "testing" (or validation) $(n=399)$ subgroup. A linear regression model was used to assess the coefficients for the included variables in the training subsets. To assure linear correlations between outcome and covariates, all covariates were also included additionally as squared variables. After backwards elimination of nonsignificant squared variables only the squared term for the total weekly exercise duration remained in the final model.

The final model derived from the training set was then used in the testing dataset to predict CRF and compare it to actual peak CRF values measured in the clinic. Pearson's 


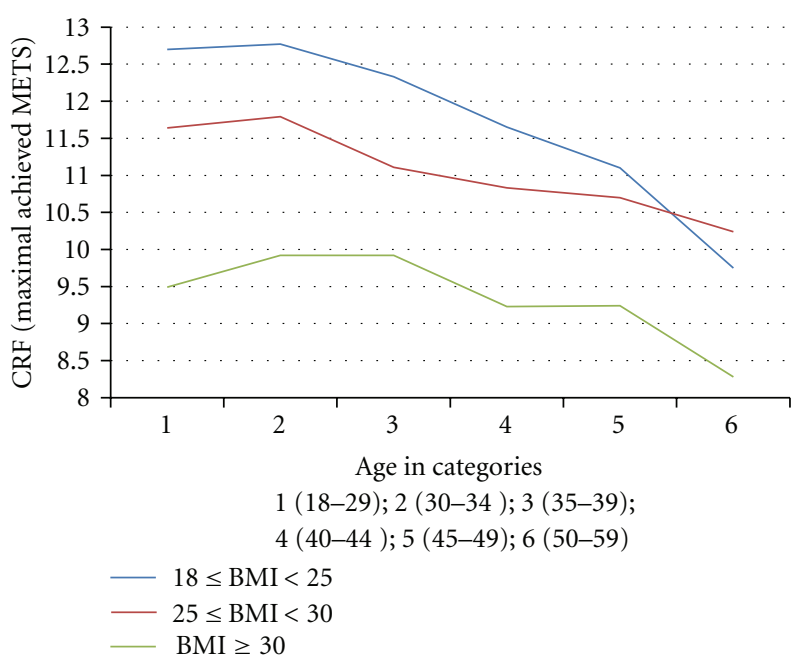

FIGURE 1: Cardiorespiratory fitness across different age categories and stratified by BMI categories.

correlation coefficient and scatter plots were used to compare the predicted values from the model with the actual observed values in the testing dataset.

Analyses were performed using SAS 9.2 (SAS Institute Inc., Cary, NC, USA). All tests presented are two-sided, and a $P$ value $<0.05$ is considered significant.

\section{Results}

Table 1 describes the baseline fitness and PA characteristics of our cohort (Table 1). Most of the firefighters are under 40 years of age, with $40 \%$ between the age of 30 and 39 . Nearly half of the study population is overweight $(25 \leq \mathrm{BMI}<30)$, and $36 \%$ are obese (BMI $\geq 30)$. The estimated total weekly exercise duration of the cohort is 102.5 minutes (SD 86.9) (Table 1).

Maximal achieved METS-across increasing age and stratified by different BMI categories-are presented in Figure 1. CRF declined over the different age categories in each of the BMI groups, though the lines for the three BMI groups are clearly separated with the normal BMI group demonstrating the highest CRF and the obese group demonstrating the lowest CRF. A similar relationship between CRF, BMI, estimated duration of weekly exercise, and different age categories is illustrated in Figure 2. The trajectory of CRF in firefighters who are obese and exercise little starts and finishes much lower in comparison to leaner colleagues who exercise more.

The predicted relationship between CRF over the career span depending on different BMI categories and weekly exercise duration pattern is illustrated in Figure 3. Even in two hypothetical situations where BMI is the same, firefighters who exercise more have less decrement in fitness levels.

The linear regression model predicting CRF in the training set of our cohort is presented in Table 2. Age, BMI, total weekly aerobic duration (singular and squared), and
TABLE 1: Baseline characteristics $(n=804)$.

\begin{tabular}{|c|c|}
\hline Age mean (SD) & $37.4(8.4)$ \\
\hline BMI mean (SD) & $29.3(4.4)$ \\
\hline CRF (maximal METS) mean (SD) & $10.7(2.0)$ \\
\hline \multicolumn{2}{|l|}{ Cardio exercise per session $n(\%)$} \\
\hline None & $44(5.5)$ \\
\hline$<15$ minutes & $74(9.3)$ \\
\hline 15-30 minutes & $340(42.8)$ \\
\hline 30-45 minutes & $228(28.7)$ \\
\hline 45-60 minutes & $67(8.4)$ \\
\hline$>60$ minutes & $41(5.2)$ \\
\hline \multicolumn{2}{|l|}{ Minutes of weight training per session $n(\%)$} \\
\hline None & $88(11.0)$ \\
\hline$<15$ minutes & $106(13.3)$ \\
\hline 15-30 minutes & $228(28.6)$ \\
\hline $30-45$ minutes & $178(22.3)$ \\
\hline 45-60 minutes & $106(13.3)$ \\
\hline$>60$ minutes & $91(11.4)$ \\
\hline \multicolumn{2}{|l|}{ Intensity of training $n(\%)$} \\
\hline Do not exercise often & $28(3.5)$ \\
\hline Light sweat & $146(18.4)$ \\
\hline Moderate sweat & $445(56.0)$ \\
\hline Heavy sweat & $176(22.1)$ \\
\hline \multicolumn{2}{|l|}{ Weekly exercise frequency $n(\%)$} \\
\hline Never & $34(4.3)$ \\
\hline 1 or less & $96(12.1)$ \\
\hline $2-3$ & $244(30.6)$ \\
\hline $3-4$ & $246(30.9)$ \\
\hline $5-6$ & $132(16.6)$ \\
\hline Every day & $44(5.5)$ \\
\hline \multicolumn{2}{|l|}{$\begin{array}{l}\text { Estimated total weekly duration mean (SD) } \\
\text { (in minutes) }\end{array}$} \\
\hline Tertile one & $30.7(23.2)$ \\
\hline Tertile two & $94.6(18.7)$ \\
\hline Tertile three & $195.2(81.3)$ \\
\hline $\begin{array}{l}\text { Estimated total weekly exercise duration } \\
\text { mean (SD) }\end{array}$ & $102.5(86.9)$ \\
\hline
\end{tabular}

BMI (body mass index), CRF (cardiorespiratory fitness), SD (standard deviation).

duration of weight lifting each week produced statistically significant $P$ values to estimate maximal achieved fitness. Every unit increase in age leads to a decline in METS of -0.05886 times age after adjusting for all the other factors in the model.

The calculated estimates were evaluated in a testing set, and the Pearson's correlation coefficient was computed to be 0.6286 . The correlation between the estimated and actual CRF is displayed in a scatter plot (Figure 4). The mean, maximum, and minimum values of the actual observed METS and of the predicted METS based on the model are shown in Table 3. 


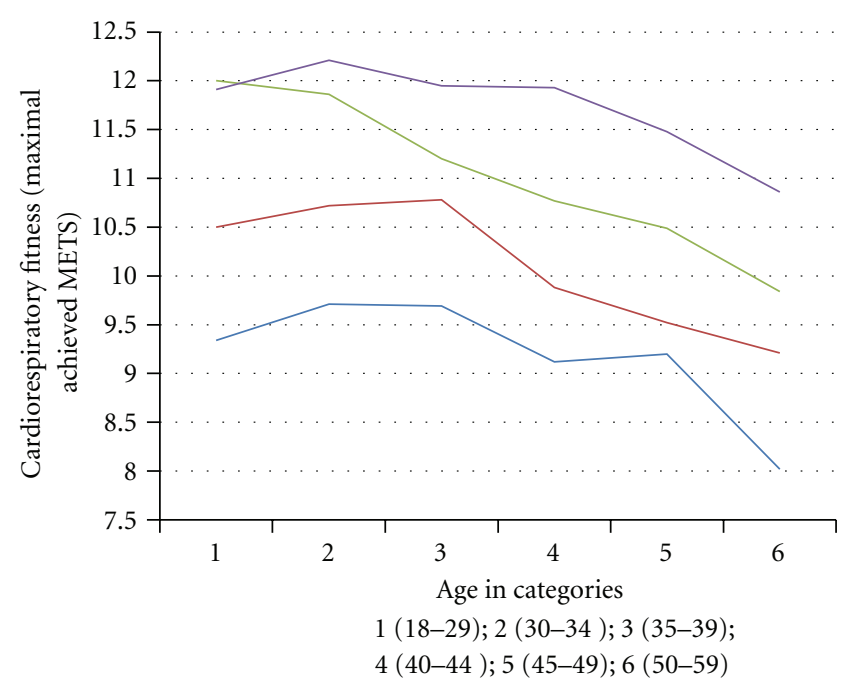

BMI $\geq 30$ and est. weekly exercise duration $<150$

- BMI $\geq 30$ and est. weekly exercise duration $\geq 150$

- BMI $<30$ and est. weekly exercise duration $<150$

$-\mathrm{BMI}<30$ and est. weekly exercise duration $\geq 150$

Figure 2: Cardiorespiratory fitness across different age categories and stratified by BMI/estimated weekly exercise duration.

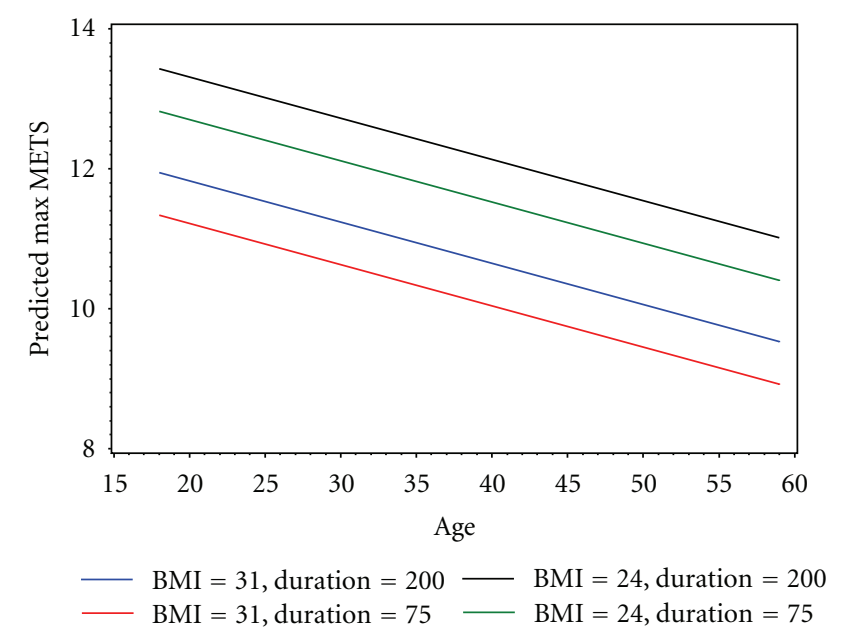

FIgURE 3: Predicted maximal Cardiorespiratory fitness (CRF) over the age span with the model calculated with the training set.

\section{Discussion}

As expected we found an age-related decline of CRF in our cohort of male career firefighters.

However, the starting, intermediate, and final points for CRF across the career span differ markedly according to the firefighters-measured BMI and self-reported physical activity. While obese firefighters had lower CRF values across the age spectrum compared to normal weight subjects (Figure 1), physical exercise attenuates the decline (Figure 2). Additionally we have demonstrated that CRF can be predicted with a high correlation coefficient including age, BMI, and variables describing physical fitness habits in the model.
TABLE 2: Linear regression model describing Cardiorespiratory fitness (maximal achieved METS) (in randomly assigned training set $n=405)$.

\begin{tabular}{lccc}
\hline Variable & Parameter estimate & Standard error & $P$ value \\
\hline Age & -0.05886 & 0.01031 & $<0.0001$ \\
BMI & -0.21131 & 0.01994 & $<0.0001$ \\
Total weekly duration & 0.00845 & 0.00232 & 0.0003 \\
$\begin{array}{l}\text { Total weekly duration } \\
\text { squared }\end{array}$ & -0.00001297 & 0.000000569 & 0.0233 \\
$\begin{array}{l}\text { Duration of weight } \\
\text { lifting }\end{array}$ & 0.18937 & 0.06393 & 0.0032 \\
Intensity of exercise & 0.21331 & 0.12362 & 0.0878 \\
\hline
\end{tabular}

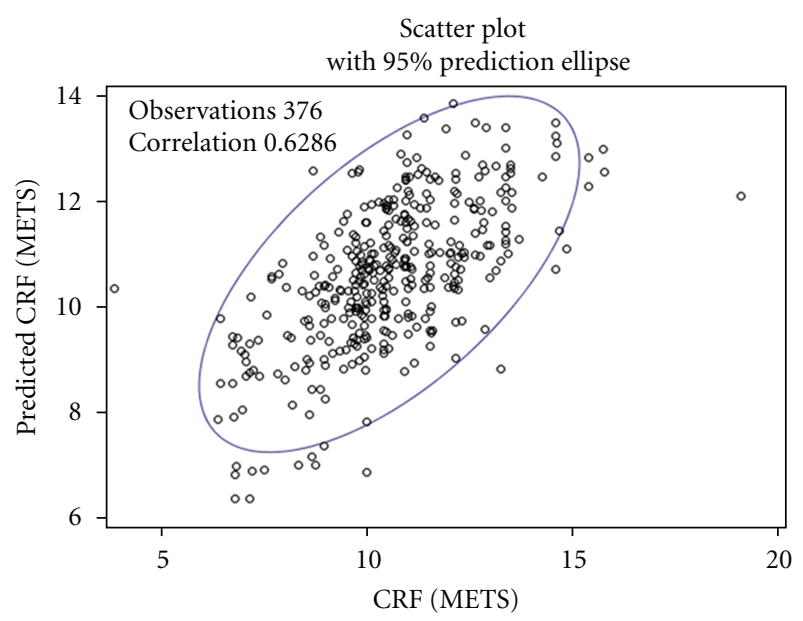

FIGURE 4: Scatter plot of predicted Cardiorespiratory fitness compared to actual values in the testing set.

TABle 3: Estimated Cardiorespiratory fitness (maximal achieved METS) and actual maximal METS in testing set $(n=399)$.

\begin{tabular}{lcccc}
\hline & $n$ & mean (SD) & Minimum & Maximum \\
\hline $\begin{array}{l}\text { Max. METS } \\
\text { Estimated } \\
\text { max. METS }\end{array}$ & 384 & $10.56363(1.87003)$ & 3.90317 & 19.01499 \\
\hline
\end{tabular}

Based on our results, albeit cross-sectional, much of the age-related decline in CRF is potentially preventable by maintaining high levels of physical activity and a healthy BMI. The ability to modify aging related declines in CRF is especially important among firefighters who are so dependent on their maximal oxygen consumption in order to safely perform their duties, and as a profession, it has cardiovascular events as the leading cause of on-duty death.

Unpredictable bouts of strenuous activity in otherwise sedentary individuals are a known precursor or acute coronary events [16], as well as periods of emotional stress [17]. Firefighters are much more likely to experience cardiovascular disease (CVD) events during periods of alarm response of fire suppression/active firefighting than during other duties at the firehouse [18]. 
In the general population the longitudinal decline in peak maximal oxygen consumption is not linear across the age span but accelerates markedly especially in men [2]. This nonlinear decline accelerates especially after 45 years of age [19]. In our cohort, especially in firefighters among higher age groups, the cross-sectional age-related decline in CRF seems to be more prominent.

4.1. Strength of the Present Study. A particular strength of the present study was the large sample size which allowed for adequate power and adjustment for confounders. BMI was calculated from measured weight and height in bare feet and light clothes during medical examinations, which avoided self-reporting biases towards lower weights and taller heights and other misclassification.

4.2. Limitations of the Present Study. Our study does have some modest limitations, including the cross-sectional design, which does not allow us to establish causation. Because of the very small number of female career firefighters, we limited our investigation to male firefighters. We have evaluated physical activity through a questionnaire, which can lead to overestimation of underlying activity levels. However, in our investigation this results in even more conservative findings, that is, would tend to overestimate PA among those with low fitness and lower the effects of PA on CRF. A minor limitation regards the use of a maximal treadmill test without expired gases using peak METS as the outcome estimates true $\mathrm{VO}_{2} \max$, while a measured $\mathrm{VO}_{2} \max$, using expired gases, would have been considered the "gold standard". Furthermore, the prediction model derived from our cohort study will have to be tested in other cohorts.

\section{Conclusion}

CRF in career male firefighters is significantly reduced with increasing age. The age-related decrements in CRF, however, are highly dependent on firefighters' BMI and physical exercise habits. Firefighters who are fitter and leaner have higher baseline CRF and attenuated CRF decrements. Our investigation supports the importance of measuring CRF, physical activity and parameters of body composition and cardiovascular health at the entry-and at regular time points-throughout a firefighter's career. This would most probably be a valuable tool in preventing the above described excessive decline in CRF with increasing age of the individual firefighter. Furthermore, especially in a profession where preservation of high fitness levels is imperative for firefighters-as well as public safety-fire departments should emphasize the maintenance of frequent physical activity as well as maintaining a healthy weight.

\section{Disclosure}

S. N. Kales has served as expert witness in medicolegal cases involving firefighters and is working under contract to revise the Heart Disease Manual of the International Association of
Fire Fighters. The other authors report no conflict of interests.

\section{Acknowledgments}

The authors would like to thank all of the participating firefighters, fire departments, the staff and clinical leadership of the clinics who examined the firefighters, Ms. Brianne Tuley, Dr. Lilly Ramphal, and the late Dr. William Patterson for their contributions to the underlying research project. This paper was supported by the Federal Emergency Management Agency (FEMA) Assistance to Firefighters Grant (AFG) program's awards EMW-2006-FP-01493 (PI: Dr. S.N. Kales), EMW-2009-FP-00835 (PI: Dr. S.N. Kales).

\section{References}

[1] L. Hakola et al., "Cardiorespiratory fitness in aging men and women: the DR's EXTRA study," Scandinavian Journal of Medicine \& Science in Sports, vol. 21, no. 5, pp. 679-687, 2011.

[2] J. L. Fleg, C. H. Morrell, A. G. Bos et al., "Accelerated longitudinal decline of aerobic capacity in healthy older adults," Circulation, vol. 112, no. 5, pp. 674-682, 2005.

[3] S. E. Brien, P. T. Katzmarzyk, C. L. Craig, and L. Gauvin, "Physical activity, cardiorespiratory fitness and body mass index as predictors of substantial weight gain and obesity: the Canadian physical activity longitudinal study," Canadian Journal of Public Health, vol. 98, no. 2, pp. 121-124, 2007.

[4] M. R. Carnethon, S. S. Gidding, R. Nehgme, S. Sidney, D. R. Jacobs, and K. Liu, "Cardiorespiratory fitness in young adulthood and the development of cardiovascular disease risk factors," Journal of the American Medical Association, vol. 290, no. 23, pp. 3092-3100, 2003.

[5] M. Hassinen, T. A. Lakka, L. Hakola et al., "Cardiorespiratory fitness and metabolic syndrome in older men and women: the Dose Responses to Exercise Training (DR's EXTRA) study, "Diabetes Care, vol. 33, no. 7, pp. 1655-1657, 2010.

[6] D. E. Laaksonen, H. M. Lakka, J. T. Salonen, L. K. Niskanen, R. Rauramaa, and T. A. Lakka, "Low levels of leisuretime physical activity and cardiorespiratory fitness predict development of the metabolic syndrome," Diabetes Care, vol. 25, no. 9, pp. 1612-1618, 2002.

[7] E. S. Soteriades, R. Hauser, I. Kawachi, D. Liarokapis, D. C. Christiani, and S. N. Kales, "Obesity and cardiovascular disease risk factors in firefighters: a prospective cohort study," Obesity Research, vol. 13, no. 10, pp. 1756-1763, 2005.

[8] S. N. Kales, G. N. Polyhronopoulos, J. M. Aldrich, E. O. Leitao, and D. C. Christiani, "Correlates of body mass index in hazardous materials firefighters," Journal of Occupational and Environmental Medicine, vol. 41, no. 7, pp. 589-595, 1999.

[9] D. M. Baur et al., "Cardiorespiratory fitness predicts cardiovascular risk profiles in career firefighters," Journal of Occupational and Environmental Medicine, vol. 53, no. 10, pp. 11551160, 2011.

[10] D. M. Baur, C. A. Christophi, and S. N. Kales, "Metabolic syndrome is inversely related to cardio-respiratory fitness in male career firefighters," The Journal of Strength \& Conditioning Research. In press.

[11] G. Durand, A. J. Tsismenakis, S. A. Jahnke, D. M. Baur, C. A. Christophi, and S. N. Kales, "Firefighters' physical activity: relation to fitness and cardiovascular disease risk," Medicine 
\& Science in Sports \& Exercise, vol. 43, no. 9, pp. 1752-1759, 2011.

[12] E. S. Soteriades, D. L. Smith, A. J. Tsismenakis, D. M. Baur, and S. N. Kales, "Cardiovascular disease in US firefighters: a systematic review," Cardiology in Review, vol. 19, no. 4, pp. 202-215, 2011.

[13] K. L. Elsner and F. W. Kolkhorst, "Metabolic demands of simulated firefighting tasks," Ergonomics, vol. 51, no. 9, pp. 1418$1425,2008$.

[14] I. Holmér and D. Gavhed, "Classification of metabolic and respiratory demands in fire fighting activity with extreme workloads," Applied Ergonomics, vol. 38, no. 1, pp. 45-52, 2007.

[15] C. Foster, A. S. Jackson, and M. L. Pollock, "Generalized equations for predicting functional capacity from treadmill performance," American Heart Journal, vol. 107, no. 6, pp. 12291234, 1984.

[16] M. A. Mittleman, M. Maclure, and G. H. Tofler, "Triggering of acute myocardial infarction by heavy physical exertion. Protection against triggering by regular exertion. Determinants of Myocardial Infarction Onset Study Investigators," The New England Journal of Medicine, vol. 329, no. 23, pp. 1677-1683, 1993.

[17] U. Wilbert-Lampen, D. Leistner, S. Greven et al., "Cardiovascular events during World Cup Soccer," The New England Journal of Medicine, vol. 358, no. 5, pp. 475-483, 2008.

[18] S. N. Kales, E. S. Soteriades, C. A. Christophi, and D. C. Christiani, "Emergency duties and deaths from heart disease among firefighters in the United States," The New England Journal of Medicine, vol. 356, no. 12, pp. 1207-1215, 2007.

[19] A. S. Jackson, X. Sui, J. R. Hébert, T. S. Church, and S. N. Blair, "Role of lifestyle and aging on the longitudinal change in cardiorespiratory fitness," Archives of Internal Medicine, vol. 169, no. 19, pp. 1781-1787, 2009. 


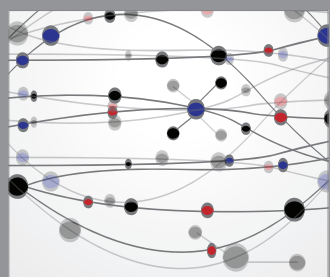

The Scientific World Journal
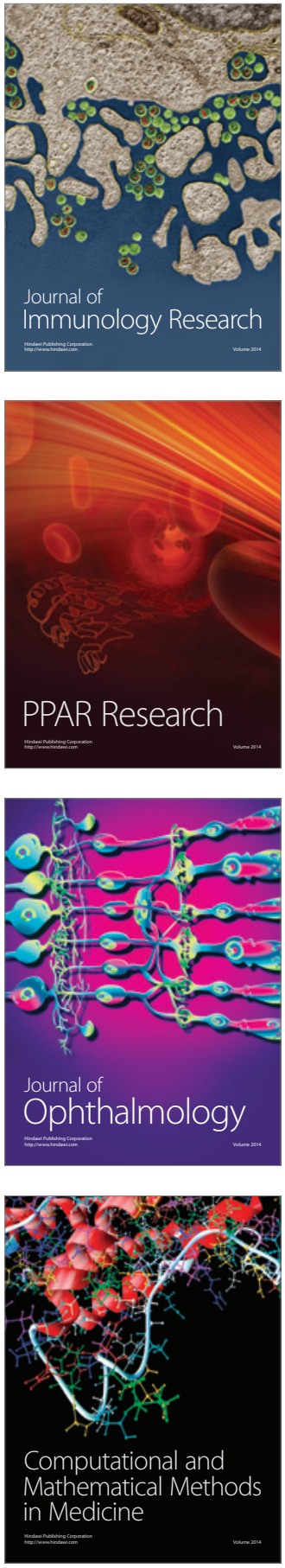

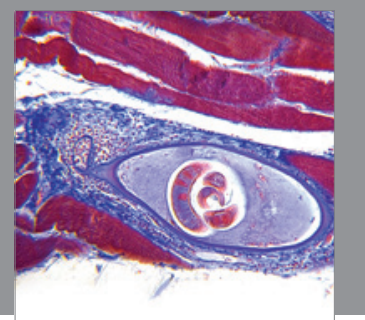

Gastroenterology

Research and Practice
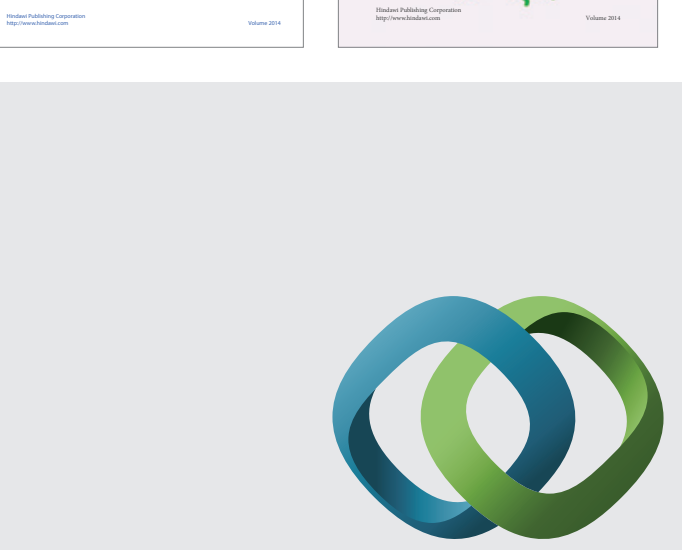

\section{Hindawi}

Submit your manuscripts at

http://www.hindawi.com
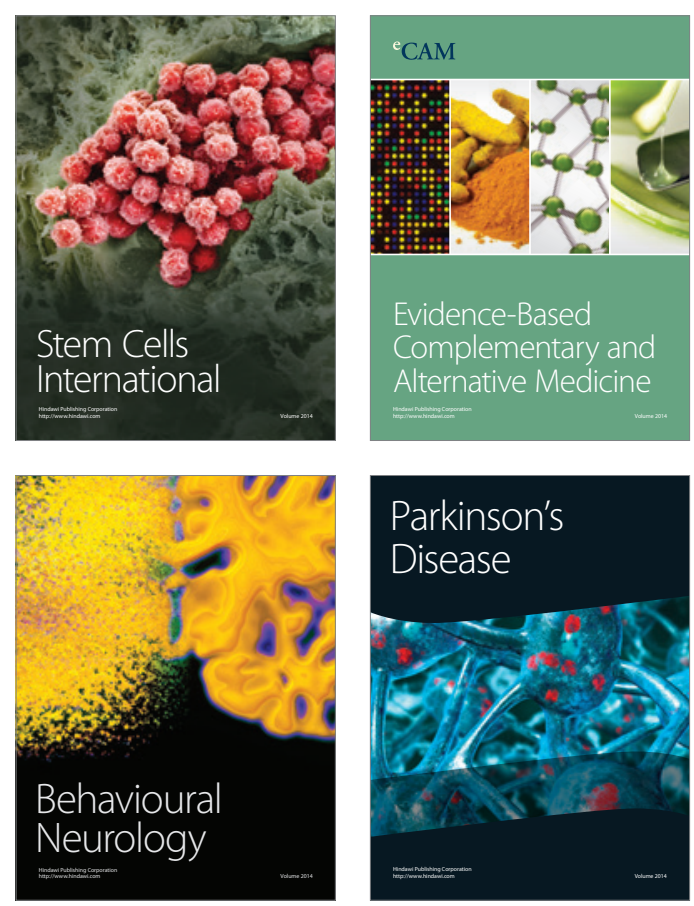

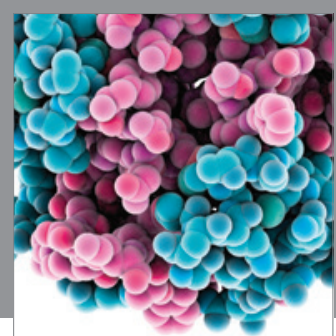

Journal of
Diabetes Research

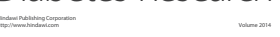

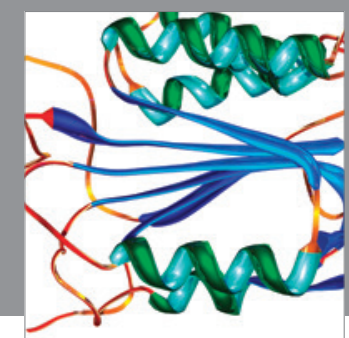

Disease Markers
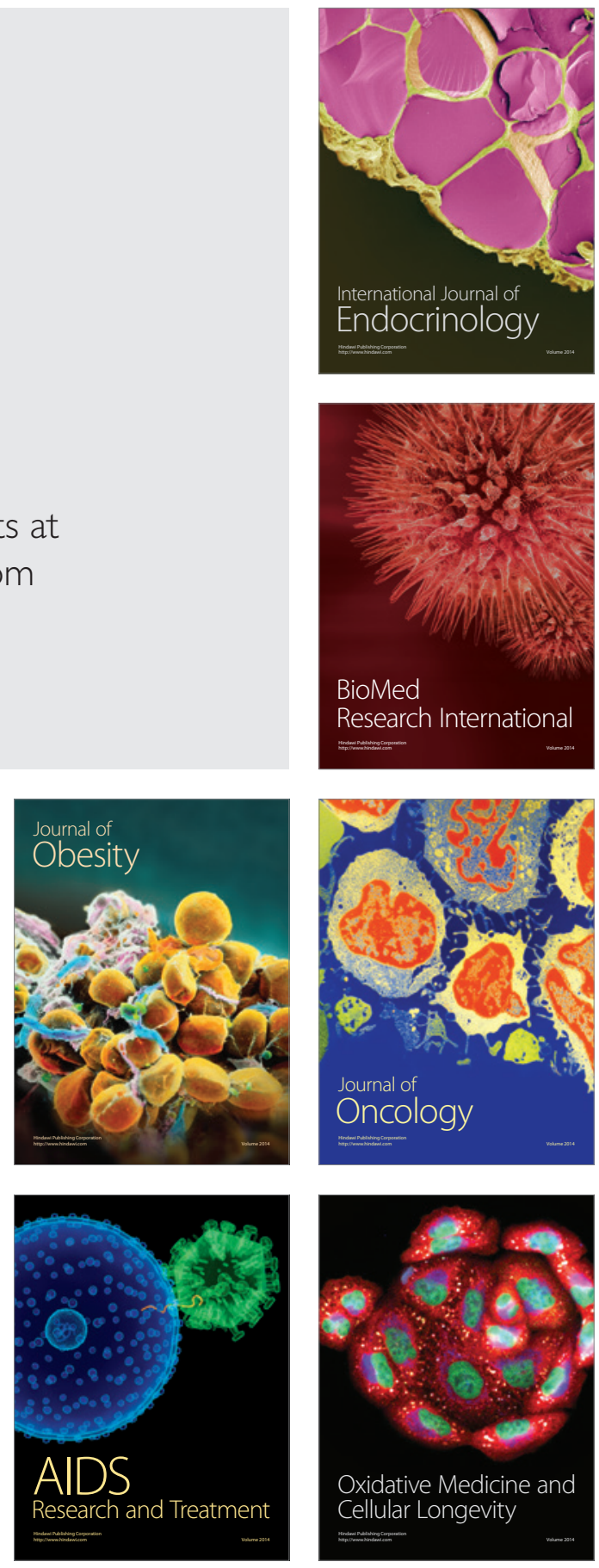